\title{
Evaluating Vertical Transmission of COVID-19 from Mothers to Neonates: An Iranian Case Series of 8 Patients
}

\author{
Shobo Sheikhahmadi, MD"; Shirin Behzadi, MD²; Avat Karimi, MD²; Farima Zakaryaei, MD $^{3^{*}}$ (D) \\ ${ }^{1}$ NICU, Besat Hospital, Sanandaj, Iran \\ ${ }^{2}$ Department of Pediatrics, Kurdistan University of Medical Sciences, Sanandaj, Iran \\ ${ }^{3}$ Department of Emergency Medicine, Kurdistan University of Medical Sciences, Sanandaj, Iran
}

\begin{abstract}
The coronavirus epidemic first broke out in China in 2019 and spread around the world. Not only the signs, symptoms, and outcomes of the coronavirus in neonates, but also its vertical transmission risks are still unknown. This case series presents eight neonates born to mothers with COVID-19 and two neonates with this infection.

Keywords: Coronavirus, Iran, Neonates

Cite this article as: Sheikhahmadi S, Behzadi S, Karimi A, Zakaryaei F. Evaluating Vertical Transmission of COVID-19 from Mothers to Neonates: An Iranian Case Series of 8 Patients. Arch Iran Med. Arch Iran Med. 2021;24(5):405-408. doi: 10.34172/aim.2021.58
\end{abstract}

Received: November 1, 2020, Accepted: February 27, 2021, ePublished: May 1, 2021

\section{Introduction}

The coronavirus disease 2019, or COVID-19, was first detected in Wuhan, China, in December 2019. ${ }^{1,2}$ Vertical transmission of coronavirus disease has not been established yet. ${ }^{3}$ The immature immune system of neonates allows for vertical transmission. Neonates that are very susceptible to infection by COVID-19 should be kept in an incubator. ${ }^{4,5}$ According to the World Health Organization (WHO) guidelines, mothers who are susceptible to or diagnosed with COVID-19 should initiate breastfeeding during the first hour after delivery. Moreover, all preterm, term, and low-birth-weight newborns should receive skinto-skin contact with their mothers based on the Infection Prevention Control (IPC) guidance. Separation may only be necessary for too ill mothers, who cannot care for their neonates. ${ }^{6}$

This study presents eight pregnant mothers with the coronavirus disease, along with their neonates, who were referred to the Besat hospital in Sanandaj, Iran, from March to May 2020.

\section{Case Reports}

Case 1

In March 2020, a 29-year-old pregnant woman, at 37-week gestational age, was admitted to the hospital with cough and fever. The primary vital signs were body temperature of $39.2^{\circ} \mathrm{C}$ and an oxygen saturation of $91 \%$. The primary test results showed lymphopenia and CRP $(2+)$ (Table 1). Her chest CT scan showed bilateral ground-glass opacity (GGO). Therefore, the patient underwent PCR for the possibility of coronavirus infection and the result was positive. After two days, she underwent normal vaginal delivery (NVD).

The newborn was a low-birth-weight girl with a 1-minute Apgar score of 2 and a 5-minute Apgar score of 4. She was intubated and transferred to the neonatal intensive care unit (NICU) for further monitoring. Due to her mother's infection, the neonate underwent a PCR test of the nasopharyngeal sample for coronavirus infection diagnosis. The PCR test result was negative. After three days, the neonate was extubated and breastfed by the mother according to the IPC guideline. Finally, she was discharged in good general health conditions.

\section{Case 2}

A 34-year-old pregnant woman, at 39-week gestational age, was admitted to the hospital due to ROM, along with a one-day history of weakness, lethargy, nausea, vomiting, and fever. The primary vital signs were body temperature of $38.7^{\circ} \mathrm{C}$ and oxygen saturation of $90 \%$. The test results showed CRP $(+)$ (Table 1). Her CT scan showed GGO in her chest. Therefore, the patient underwent PCR for the possibility of coronavirus infection and her test result was positive. She underwent a cesarean section, due to prior cesarean delivery.

The newborn was a normal-birth-weight girl with a 1-minute Apgar score of 9 and a 5-minute Apgar score of 10. The neonate was monitored because of her infected mother; however, they were not separated and she was breastfed in compliance with the IPC guideline. The neonate had no COVID-19 symptom and her PCR test result was negative. After five days, she was discharged in 
Table 1. Primary Laboratory Results of Mothers

\begin{tabular}{lccccccc}
\hline & $\mathbf{W B C}(\boldsymbol{\mu L})$ & Neut $\%$ & Lymph $\%$ & $\mathbf{H b}(\mathbf{g} / \mathbf{d L})$ & $\mathbf{P l t}(/ \boldsymbol{\mu L})$ & CRP & ESR $(\mathbf{m m} / \mathbf{h})$ \\
\hline Case 1 & 4500 & 90 & 10 & 12 & 210000 & $2+$ & 33 \\
Case 2 & 11100 & 70 & 30 & 9.9 & 363000 & $1+$ & 23 \\
Case 3 & 11000 & 70 & 30 & 11 & 150000 & NEG & 30 \\
Case 4 & 7500 & 85 & 15 & 12 & 225000 & $2+$ & 32 \\
Case 5 & 9000 & 85 & 15 & 11 & 270000 & $1+$ & 39 \\
Case 6 & 8000 & 83 & 17 & 10.5 & 310000 & $2+$ & 34 \\
Case 7 & 14200 & 80 & 20 & 12 & 155000 & $1+$ & 31 \\
Case 8 & 8000 & 90 & 10 & 10 & 185000 & $1+$ & 35 \\
\hline
\end{tabular}

good general health conditions.

\section{Case 3}

A 25-year-old pregnant woman, at 33-week gestational age, was admitted to the hospital due to polyhydramnios and the onset of contractions, along with a two-day history of cough and dyspnea. The primary vital signs were body temperature of $37.8^{\circ} \mathrm{C}$ and an oxygen saturation of $91 \%$. The test results showed lymphopenia (Table 1). Her CT scan result was normal while the PCR result was positive for the coronavirus. Therefore, she underwent a cesarean section.

The newborn was a low-birth-weight preterm boy with a 1-minute Apgar score of 5 and a 5-minute Apgar score of 7 . He was intubated because of the intensification of distress and low oxygen saturation. He was transferred to the NICU because of distress and abdominal ascites, with the diagnosis of neonatal hydrops. Since his mother was positive for COVID-19, he underwent the PCR test for possible infection. Although his PCR result was negative for COVID-19, he died from respiratory distress after seven days.

\section{Case 4}

A 27-year-old pregnant woman, at 28-week gestational age, was admitted to the hospital due to the onset of contractions, along with a two-day history of cough and fever. The primary vital signs were body temperature of $38.5^{\circ} \mathrm{C}$ and an oxygen saturation of $93 \%$. The test results showed lymphopenia and CRP (2+) (Table 1). Her CT scan showed bilateral GGO in her lungs. Therefore, the patient underwent PCR for the possibility of coronavirus infection and the result was positive. Therefore, she underwent NVD.

The newborn was a low-birth-weight preterm boy with a 1-minute Apgar score of 3 and a 5-minute Apgar score of 5 . He was transferred to the NICU because of distress and preterm conditions. He was then intubated for intensification of distress and low oxygen saturation. Since his mother was infected with COVID-19, he underwent the PCR test for possible infection and the test result was positive. However, he died after five days because of severe neonatal respiratory distress.
Case 5

A 27-year-old pregnant woman, at 39-week gestational age, was admitted to the hospital due to the onset of contractions, along with a three-day history of cough. The primary vital signs were body temperature of $38.5^{\circ} \mathrm{C}$ and an oxygen saturation of $91 \%$. The test results showed CRP (+) (Table 1). Her chest CT scan was normal while the PCR test was positive for the coronavirus. Therefore, she underwent NVD.

The newborn was a normal-birth-weight term boy with a 1-minute Apgar score of 9 and a 5-minute Apgar score of 10 . The neonate was monitored because of his mother's infection; however, they were not separated and he was breastfed by his mother according to the IPC guideline. No signs and symptoms of the coronavirus disease were observed in him. Since his PCR test was negative for COVID-19 disease, he was discharged after 7days in good general health conditions.

\section{Case 6}

A 30-year-old pregnant woman, at 36-week gestational age, was admitted to the hospital due to the onset of contractions. She underwent NVD.

The newborn was a normal-birth-weight term girl with a 1-minute Apgar score of 9 and a 5-minute Apgar score of 10. The neonate's condition allowed her to room in with her mother and was breastfed. However, the mother started a high-grade fever $\left(\mathrm{Temp}=39.3^{\circ} \mathrm{C}\right)$ two hours after the delivery. The test results showed lymphopenia and CRP (2+) (Table 1). Her chest CT scan showed GGO. Therefore, the PCR test of the nasopharyngeal sample was performed for the mother and the neonate.

They were not separated and she was breastfed by her mother according to the IPC guideline. The neonate was kept under monitoring. No signs and symptoms of COVID-19 were observed in the neonate.

The PCR test of the mother was positive for the coronavirus disease; however, her neonate's result was negative. The neonate was discharged after seven days in good general health conditions.

\section{Case 7}

A 34-year-old pregnant woman, at 34-week gestational age, was admitted to the hospital due to headache and 
hypertension $(\mathrm{SBP}=170)$. Due to preeclampsia, she underwent an emergency NVD.

The newborn was a low-birth-weight preterm girl with a 1-minute Apgar score of 2 and a 5-minute Apgar score of 4. She was intubated and transferred to the NICU because of distress and preterm conditions.

The mother started a low-grade fever one hour after delivery. Her test results showed lymphopenia and CRP $(+)$ (Table 1). Her chest CT scan showed bilateral GGO in her lungs. The neonate's test result also showed CRP (+) (Table 2). As a result, the PCR test of the nasopharyngeal sample was performed for the mother and her neonate.

The PCR tests of the mother and her neonate were positive for the coronavirus. As a result, the neonate was transferred to the NICU and isolated; however, she was breastfed by her mother according to the IPC guideline. Moreover, she underwent antibiotic therapy with ampicillin and gentamycin. She was discharged in good general health conditions.

\section{Case 8}

A 30-year-old pregnant woman, at 39-week gestational age, was admitted to the hospital due to the onset of contractions. Therefore, she underwent NVD. She had a history of addiction. Her primary test results showed lymphopenia and CRP (+) (Table 1). No signs and symptoms of COVID-19 were observed in the patient.

The newborn was a normal-birth-weight term boy with a 1-minute Apgar score of 6 and a 5-minute Apgar score of 9. Because of her mother's addiction and neonatal distress, he was transferred to the neonatal unit. In addition, his test results showed CRP (+) (Table 2).

Since the mother was highly susceptible, she and her neonate underwent a PCR test for coronavirus infection diagnosis. The PCR tests of the mother and her neonate were positive for coronavirus. As a result, the neonate was transferred to the neonatal unit and isolated; however, he was breastfed by her mother according to the IPC guideline. Moreover, he underwent antibiotic therapy with ampicillin and gentamycin. He was discharged after two weeks in good general health conditions.

Two-week post-discharge follow-up was planned for all infected neonates. The neonates underwent physical and clinical examinations and their nasopharyngeal samples were examined using the PCR. The test results were normal and the PCR tests were negative for the coronavirus infection.

\section{Discussion}

This study presented eight pregnant women admitted to the Be'sat Hospital in Sanandaj, Iran, from March to May
2020. All eight women were in their third trimester and infected with the coronavirus.

The patients had no history of medical diseases and one of them had a history of addiction. Their common signs and symptoms were fever, cough, high body temperature, and low oxygen saturation. The mean duration of the pre-admission symptom was one day. Based on the test results, lymphopenia was observed in five patients and seven patients were observed with an increased level of CRP. Moreover, five participating mothers had chest CT abnormalities.

According to a recent study, pregnant women infected with COVID-19 may show fewer symptoms. In addition, a higher rate of preterm birth, low birth weight, C-section, and NICU admission was observed in this group. ${ }^{7}$ Four participants had preterm labor, two out of the noninfected neonates died from neonatal hydrops and severe distress syndrome.

Although all the neonates were born to mothers in the acute phase of the disease, only two of them were diagnosed with SARS-CoV-2 infection. These two neonates were given birth through the NVD and both stayed alive. It is expected that the risk of vertical transmission is high in NVD because of the contact between the neonate's upper respiratory tract and the mother's anus. Therefore, it should be established whether SARS-CoV-2 can transmit vertically through the placenta or not. ${ }^{8}$ It has been found that the SARS-CoV-2 in the neonates' upper respiratory tracts or anuses have maternal origin. ${ }^{9}$ Some recent studies reported no SARS-CoV-2 in the amniotic fluid, cord blood, or breast milk samples. ${ }^{10}$

In the present study, although the infected neonates with SARS-CoV-2 had respiratory distress, none of them was observed to have fever, poor feeding, vomiting, or diarrhea. According to the findings of Zeng et al, neonates are more symptomatic from prematurity, asphyxia, and sepsis, than the SARS-CoV-2 infection. ${ }^{9}$ Dumpa et al reported a horizontal infection in a neonate, whose main symptom was fever. ${ }^{11}$ Kamali et al reported that fever and a positive history of COVID-19 in parents are important factors in a neonate with coronavirus infection. ${ }^{12}$

The only treatments the infected neonates received were supportive management and antibiotic therapy. A recent study showed that antiviral and anti-cytokine drugs are not useful for treating children with COVID-19. For these patients, such supportive management strategies as respiratory support and monitoring, blood examinations, and chest X-ray are recommended. ${ }^{4}$

In conclusion, the results showed that COVID-19 has good prognosis in neonates with no congenital problem. It is recommended to use appropriate supportive

Table 2. Infected Neonates' Laboratory Results

\begin{tabular}{lccccc}
\hline & WBC $(\boldsymbol{\mu L})$ & Lymph $\%$ & Neut $\%$ & Hb(g/dL) & Plt $(/ \mu L)$ \\
\hline Case 7 & 11800 & 80 & 20 & 13 & 150000 \\
Case 8 & 6600 & 30 & 70 & 11.5 & 580000 \\
\hline
\end{tabular}


management and antibiotic therapy for neonates infected with COVID-19. The participants were treated without using any antiviral drugs. In conclusion, it is suggested that NVD can increase the risk of viral transmission due to the contact between the neonate's upper respiratory tract and the mother's anus.

\section{Authors' Contribution}

SS designed the study and critically reviewed the manuscript. $\mathrm{FZ}$ was the major contributor in writing the manuscript; AK and SB collected the data; all authors read and approved the final manuscript.

\section{Conflict of Interest Disclosures}

The authors declare that they have no conflict of interest.

\section{Ethical Statement}

This manuscript has been ethically approved by the ethics committee of Kurdistan University of Medical Sciences, Sanandaj, Iran. (ID:IR.MUK.REC.1399.300)

\section{References}

1. Cascella M, Rajnik M, Cuomo A, Dulebohn SC, Di Napoli R. Features, Evaluation, and Treatment of Coronavirus (COVID-19). StatPearls. Treasure Island (FL): StatPearls Publishing; 2021.

2. Zhu N, Zhang D, Wang W, Li X, Yang B, Song J, et al. A novel coronavirus from patients with pneumonia in China, 2019. N Engl J Med. 2020;382(8):727-33. doi:10.1056/ NEJMoa2001017

3. Duran P, Berman S, Niermeyer S, Jaenisch T, Forster T, Gomez Ponce de Leon R, et al. COVID-19 and newborn health: systematic review. Rev Panam Salud Publica. 2020;44:e54. doi:10.26633/RPSP.2020.54

4. De Rose DU, Piersigilli F, Ronchetti MP, Santisi A, Bersani I, Dotta A, et al. Novel Coronavirus disease (COVID-19) in newborns and infants: what we know so far. Ital J Pediatr.

\section{0;46(1):56. doi:10.1186/s13052-020-0820-x}

5. Li F, Feng ZC, Shi Y. Proposal for prevention and control of the 2019 novel coronavirus disease in newborn infants. Arch Dis Child Fetal Neonatal Ed. 2020;105(6):683-684. doi:10.1136/archdischild-2020-318996

6. World Health Organization. Clinical management of severe acute respiratory infection (SARI) when COVID-19 disease is suspected: interim guidance, 13 March 2020. World Health Organization; 2020.

7. Smith V, Seo D, Warty R, Payne O, Salih M, Chin KL, et al. Maternal and neonatal outcomes associated with COVID-19 infection: A systematic review. PLoS One. 2020;15(6):e0234187. doi: 10.1371/journal.pone.0234187

8. Wang S, Guo L, Chen L, Liu W, Cao Y, Zhang J, et al. A case report of neonatal 2019 coronavirus disease in China. Clin Infect Dis. 2020;71(15):853-7. doi:10.1093/cid/ciaa225

9. Zhu H, Wang L, Fang C, Peng S, Zhang L, Chang G, et al. Clinical analysis of 10 neonates born to mothers with 2019-nCoV pneumonia. Transl Pediatr. 2020;9(1):51-60. doi:10.21037/tp.2020.02.06

10. Chen H, Guo J, Wang C, Luo F, Yu X, Zhang W, et al. Clinical characteristics and intrauterine vertical transmission potential of COVID-19 infection in nine pregnant women: a retrospective review of medical records. Lancet. 2020;395(10226):809-815. doi: 10.1016/ S0140-6736(20)30360-3. Epub 2020 Feb 12. Erratum in: Lancet. 2020;395(10229):1038. Erratum in: Lancet. 2020;395(10229):1038.

11. Dumpa V, Kamity R, Vinci AN, Noyola E, Noor A. Neonatal coronavirus 2019 (COVID-19) infection: a case report and Review of Literature. Cureus. 2020;12(5):e8165. doi: $10.7759 /$ cureus.8165.

12. Kamali Aghdam M, Jafari N, Eftekhari K. Novel coronavirus in a 15-day-old neonate with clinical signs of sepsis, a case report. Infect Dis (Lond). 2020;52(6):427-9. doi: $10.1080 / 23744235.2020 .1747634$. 